\title{
Conflictos en ética de investigación con seres humanos
}

\author{
Ethical conflicts in research \\ with human subjects
}

\footnotetext{
1 Universidad de Chile, Santiago, Chile.

Correspondencia M. H. Kottow Facultad de Filosofía y Humanidades, Universidad de Chile. Casilla 16168, correo 9, Santiago, Chile. kottow@terra.cl

mkottow@med.uchile.cl
}

\begin{abstract}
The Declaration of Helsinki (2000) helped trigger a major debate on many ethical aspects of research with human subjects. Topics under discussion include the use of placebos, the application of equipoise, the distinction between research ethics and clinical ethics, and the difference between therapeutic and non-therapeutic trials. This article supports tenets that serve to protect patients involved in research, especially in those aspects concerning maintenance of best existing therapy, the rejection of using placebos, support of equipoise, the assurance that required medication shall be available beyond the termination of the research project, and that appropriate compensation will obtain to host communities inasmuch as they may have facilitated and supported the project. The current proposal of introducing a double ethical standard, aspirational for developed countries and pragmatic for poor nations, should be rejected, since it contravenes the universalizability of prescriptive ethics.
\end{abstract}

Research, Ethics; Helsinki Declaration; Placebos

\section{Introducción}

El Código de Nüremberg (1947) constituyó el hito fundador de la ética de investigación con seres humanos, además de inaugurar un estilo normativo que marcó el desarrollo de las Declaraciones de Helsinki. Todos estos documentos se han inspirado en un clima de desasosiego y preocupación gestado por prácticas de investigación notoriamente defectuosas que debían ser eliminadas y reemplazadas por prescripciones que corrigiesen las deficiencias prevalentes. Nüremberg fue la reacción a las atrocidades del nacionalsocialismo alemán (19331945), en tanto Helsinki y los encuentros posteriores de la Asociación Médica Mundial (World Medical Association) - ocurrieron en un clima de transgresiones a la ética de investigación: las denunciadas por Beecher en 1966, las desastrosas investigaciones de Willowbrook - iniciadas en 1956 -, Tuskegee Valley 1932-1972, Hospital Judaico de Enfermedades Crónicas comentado en Science 1964. Así, se explica que Helsinki ha debido ser revisada en cinco oportunidades, en relación con nuevas transgresiones éticas que no habían sido previamente detectadas. Para Brody 1 (p. 53), las "políticas [nacionales e internacionales en torno a la investigación en seres humanos] emergieron en respuesta tanto a las atrocidades de la II Guerra Mundial como a las posteriores revelaciones de serios problemas con la ética de investigación 
que involucran a sujetos humanos". Estas declaraciones, las directivas de diversas instituciones internacionales, así como el debate bioético, han desarrollado una extensa discusión sobre la ética de la investigación en seres humanos, produciendo un importante cuerpo normativo e incluso legislativo, un esfuerzo que en Latinoamérica ha sido liderado por Brasil 2.

La Declaración de Helsinki (Endiburgh, 2000) se destaca de las anteriores por establecer estándares éticos prospectivos: comenta y establece las prácticas éticamente inaceptables, pero también propone corregir para el futuro ciertas estrategias tradicionalmente utilizadas en la investigación. Diversas exigencias al método de investigación, así como ciertos elementos prospectivos de Helsinki 2000, desencadenaron de forma inédita reacciones de rechazo y la demanda de revisión por las naciones del primer mundo que, afectadas en sus intereses materiales, se disponían a diseñar una nueva ética de investigación. El primer impulso fue requerir enmiendas que flexibilizaran los artículos 29 y 30 , una táctica reforzada con el cuestionamiento y finalmente un intento de desprestigio de Helsinki como regulador ético ${ }^{3}$. Al desencadenarse la controversia, Helsinki perdió el ascendiente moral del que había gozado previamente y comenzaron a aparecer voces que desacreditaban la declaración, criticándola por ser una recomendación no vinculante, insuficientemente determinante 3 , producto de una "opinión minoritaria” 4 (p. 191). Todas estas críticas cometen la falacia retórica ex concessis de descalificar a quien emite una opinión, en vez de argumentar sobre el contenido de lo expresado.

La campaña de debilitamiento de Helsinki 2000 ha permitido plantear un supuesto consenso acerca de la invalidez de lo propuesto en Edinburgh. Así, se lee que "la Declaración de Helsinki ha perdido su autoridad moral" porque sus "normas carecen de justificación y elaboración de sus posiciones" 4 (p. 192); que la "Declaración de Helsinki...es simplemente una 'declaración' que los EE.UU. han estado ignorando" 3 (p. 23). Las falacias de esta posición fueron debidamente identificadas 5 , pero cometiendo el mismo error de descalificar a las instituciones consensuantes (Council for International Organizations of Medical Sciences CIOMS, The Joint United Nations Programme on HIV/AIDS - UNAIDS, National Bioethics Advisory Commission - NBAC, European Group on Ethics in Science and New Technologies EGE, Nuffield Council), una estrategia que insi- núa una pugna de poder antes que un enfrentamiento de argumentos. En efecto, a tenor de la discusión se facilita la hegemonía del más poderoso, apoyando la idea cada vez más prevalente en los países desarrollados de la insuficiencia de Helsinki y haciendo difícil modificar las estrategias de investigación de esas naciones. La bioética que se está desarrollando en Latinoamérica ha sido muy crítica de los intentos y de la forma de modificar Helsinki 2000, aunque tiene escasa oportunidad de contrargumentar con efectividad en los escenarios de primer mundo, siendo aconsejable replegarse al escenario local y reforzar allí las capacidades de resistencia a la colonización científica y empresarial, en una tarea de protección a la población que es reclutada para investigaciones éticamente cuestionables 6,7,8,9.

Helsinki 2000 fue un desencadenante; sin embargo, el conflicto ético en torno a las investigaciones con seres humanos es mucho más complejo que la mera revisión de los dos artículos de la Declaración de Helsinki que están en polémica, llevándose en tres planos simultáneamente: conceptual, metódico, y pragmático.

\section{Conflictos conceptuales}

Declaraciones de Helsinki anteriores a la actual habían establecido la substancial diferencia, en estudios con seres humanos, entre investigaciones terapéuticas y no terapéuticas. Con mayor rigor aún, es preciso distinguir primero entre ensayos clínicos y no clínicos, para luego hacer la diferencia entre los clínicos terapéuticos y los clínicos no terapéuticos. Las investigaciones no clínicas - o pre-clínicas - se llevan a cabo en probandos sanos, para los cuales no hay beneficios, pero que están expuestos a riesgos supuestamente sostenibles y predecibles en base a los estudios previos, lo cual es asimismo válido para las investigaciones de fase I que "utilizan sujetos voluntarios normales" 10 (p. 103). La utilidad de estos ensayos es limitada, pero necesariamente previa a los estudios fase II y III en que se investiga efectos terapéuticos en pacientes. Si bien estos estudios preclínicos tienen una transparencia ética aceptable, no reemplazan a las investigaciones clínicas, más complejas en su diseño y que son el verdadero motivo de las polémicas éticas desencadenadas. Sin embargo, como los ensayos no clínicos - también denominados "experimentos cognitivos” 11 (p. 445) - implican algún 
riesgo para los probandos, no parece algo decidido que se les niegue todo beneficio compensatorio a los que participan y, eventualmente, a la comunidad de donde provienen.

Las investigaciones clínicas ocurren, por definición, en probandos que están enfermos, por lo tanto tienen una susceptibilidad aumentada y mayores riesgos de efectos negativos frente a cualquier sustancia que reciban. Si el fármaco estudiado se relaciona con la enfermedad del paciente-probando - "subjects/patients" 1 (p. 17) -, se trata de una investigación clínica terapéutica. En caso de analizar un agente cuyos efectos no están relacionados con la afección de los probandos, se considera una investigación clínica no terapéutica realizada a pacientes-probandos, es decir, en sujetos enfermos, ya vulnerados. Estas distinciones son fundamentales para entender el desacuerdo de Helsinki con gran parte de los investigadores primermundistas.

Según Helsinki, el paciente que ingresa a un estudio debe continuar recibiendo todos los cuidados médicos y los medios terapéuticos que requiera por su afección. En otras palabras, la ética clínica de cuidados al paciente debe ser respetada de forma invariable, sea o no reclutado para una investigación. Los investigadores se expresan claramente en contra de esta opinión, indicando que los rigores del método científico no deben ser debilitados con consideraciones clínico-terapéuticas. Por lo tanto, aducen, es preciso distinguir claramente la diferencia entre ética clínica y ética de investigación, para permitir a los estudios albergarse en ésta y desentenderse de aquella: “cualesquiera sean los motivos del investigador, los pacientes voluntariamente reclutados corren el riesgo de ver comprometido su bienestar en el curso de la investigación científica" 12 (p. 21). Además, se proponen eliminar la distinción que Helsinki hace entre investigaciones terapéuticas y no terapéuticas, porque para la investigación el probando no es un paciente, la pureza científica no necesariamente tiene por meta estudiar si el paciente se beneficia médicamente. Un ensayo científico analiza los efectos de una droga sobre un organismo, que podrán ser terapéuticos o no, sin que eso afecte la metódica del estudio. El ingreso de pacientes en una investigación terapéutica significa inevitablemente el perjuicio de perder los mejores cuidados médicos y quedar a merced del método científico y en la desprotección de ser asignado al grupo control. Para algunos científicos prominentes, la investigación debe aceptar que es de relevancia secundaria que la ética clínica exija proteger a los pacientes: "la investigación clínica, en contraste [con cuidados médicos]...no es una actividad terapéutica dedicada al cuidado personal de pacientes" 12 (p. 20). Esta ética de investigación se contrapone a los esfuerzos que efectúan los médicos tratantes por no ceder en su compromiso de dar los mejores cuidados terapéuticos a sus pacientes reclutados, a despecho de las intenciones científicas, y eventualmente desaconsejando su inclusión cuando ello pudiese desmedrar la atención médica requerida.

\section{Conflictos metódicos}

Lo antedicho sienta las bases para discutir tres estrategias de investigación que han estado en el centro de recientes polémicas, dos de ellas a través de la Declaración de Helsinki.

\section{Placebos}

El nombre de placebos es erróneo en el contexto de ensayos clínicos, porque no son substancias inertes dadas para complacer al paciente, sino que tienen por objeto crear la confusión del doble ciego o doble enmascarado, en la que los probandos no saben si reciben la sustancia en estudio o un substituto inerte. El único complacido es el investigador, quien queda en la favorable posición de obtener fácilmente algún resultado positivo porque el grupo control quedará sin efectos. Sin embargo, la técnica genera un doble riesgo: si el ensayo es clínico no terapéutico, los pacientes-probandos que reciban la droga en estudio están expuestos a interacciones insospechadas entre la substancia y su enfermedad, o entre aquella y la medicación que estén recibiendo. Si, en cambio, se está ante un estudio terapéutico, el grupo control quedará privado de los efectos benéficos que la nueva droga está prometiendo. El período de desprotección puede prolongarse en aquellos estudios terapéuticos que requieren una suspensión inicial wash out - de la medicación - psicofármacos, por ejemplo - que el paciente estaba recibiendo.

Todos estos efectos negativos han sido reconocidos al punto que los países desarrollados desincentivan el uso de placebos en sus propias poblaciones cuando ello priva a los probandos de un terapia eficaz y probada, pero los aprueban y utilizan profusamente en naciones del tercer mundo. En una aclaratoria posterior a la declaración del año 2000, la Asociación Médica Mundial permite el uso de placebos en dos situaciones: si no hay riesgos mayores o daños irreversibles para los probandos, y si hay motivos científicos sólidos para el uso de placebos, lo cual constituye una flexibilización que recupera nuevamente el uso de placebos con argu- 
mentos contingentes. La propuesta de enmienda presentada originalmente por Levine (apud Rothman 13, p. 62) es aún más drástica: “cuando el resultado no es la muerte ni una discapacidad, el uso de placebos u otras formas de control no terapéutico puede justificarse en base a su eficiencia". Estas excepciones son impugnables, pues siempre hay daño si se reemplaza un tratamiento reconocido como el mejor existente o como el estándar terapéutico del momento, por una substancia inerte y, en lo referente al método científico, siempre es construible el uso de placebos para mantener la pureza del ensayo, pero eso por si solo no le da validez ética; todo lo contrario, da prioridad a la experimentación científica por encima del cuidado del ser humano enfermo. En cuanto al uso de placebos en el estudio de substancias de importancia terapéutica marginal, es éticamente cuestionable que se inviertan recursos en estudios triviales y se sometan a riesgos, aunque sean menores, a pacientes que ya tienen una carga importante de riesgos por su enfermedad, y que en nada se beneficiarán por participar en estos protocolos. Es una falacia insistir que los placebos sean preferibles porque acortan precozmente los estudios y reducen los riesgos a los probandos, pues obviamente estarían mejor protegidos con la mejor terapia existente probada que con material inactivo. Además, si se quiere comparar cuán efectiva es una droga de menor costo que el tratamiento establecido, lo lógico es comparar ambas substancias en las dosis cuestionadas y no fundamentarse en artificios de cálculo para utilizar placebos 14 .

La enmienda comentada demuestra, también, que las declaraciones de Helsinki son perfeccionables, pero que ello debiera ocurrir hacia un mayor rigor y no, como se sugiere desde el primer mundo, hacia la vaguedad y la flexibilidad.

\section{Sub-medicación}

Muchos protocolos se diseñan para comparar una nueva substancia con lo actualmente en uso, situación que puede hacer difícil obtener diferencias significativas a favor del fármaco ensayado. Los investigadores han discurrido el uso de medicación sub-dosificada en comparación al estándar aceptado y que se contrasta con un placebo, lo cual hará resaltar mejor las diferencias con la droga ensayo, pero somete a los probandos a todos los efectos negativos de terapias incompletas, como resistencia del agente causal, cronicidad del cuadro parcialmente curado, tendencia a la recidiva, taquifilaxis medicamentosa. Por ello, también esta estrategia de investigación ha sido expresamente recha- zada por Helsinki al exigir que los grupos controles reciban los mejores métodos probados y existentes. Críticas de estudios como el de Mombasa, Kenya en personas HIV positivo que no recibieron tratamiento antiviral señalan que "un tal estudio no se podría haber realizado en los EE.UU. por cuanto retiene un tratamiento reconocidamente efectivo" 13 (p. 62).

En cierto modo fue la utilización de sub-medicación la que desencadenó gran parte de las discrepancias en torno al uso de placebos y tratamientos parciales. Durante un tiempo pareció ser una práctica de rutina estudiar el efecto de dosis parciales de medicación con AZT en mujeres embrazadas HIV positivo, aun cuando ensayos concluyentes habían detallado la terapia óptima - protocolo 076 - para reducir la transmisión vertical de HIV neonatal. Las voces que se levantaron en contra de estas prácticas inmorales fueron minoritarias y no han logrado revertir el uso de estas prácticas de investigación 15,16,17,18.

\section{Equiponderación (= equipoise)}

El concepto fue introducido por Fried 19 para proteger a los pacientes de investigaciones redundantes. Sólo en circunstancias clínicas con terapias alternativas aparentemente equivalentes o donde hay incertidumbres sobre el valor de una sobre otra, se da la situación de equiponderación o equipoise, que justifica o requiere un estudio para decidir la mejor opción médica. Freedman ${ }^{20}$ prefirió hablar de equiponderación clínica para apoyar estudios adicionales cuando la comunidad médica discrepaba en la interpretación de evidencia disponible, lo cual también tiene por objetivo proteger a los pacientes de tratamientos inadecuados. La controversia actual se resume en la posición de quienes hacen valer la ética clínica junto a la ética de investigación, frente a los que desean que los estudios se ciñan solo por la ética de investigación, pues los "estudios randomizados y controlados no están diseñados para, y pueden entrar en conflicto con, el cuidado [médico] personal" 12 (p. 19). Una posición difícil de aceptar desde la perspectiva de una bioética preocupada por investigaciones en poblaciones vulnerables y susceptibles, y que no está dispuesta a sacrificar la estrategia de equiponderación y descartarla porque se trataría de “un concepto turbio" 21 (p. 350).

Helsinki no ha opinado sobre equiponderación, pero no obstante también aquí hay acerbas discusiones cuya médula no es metodológica, sino que ética. La equiponderación, desarrollada por Fried 19 y por Freedman 20, se re- 
fiere a situaciones clínicas en que existen alternativas terapéuticas consideradas equivalentes en los beneficios y/o efectos negativos que producen, y en que un programa de investigación se propone dirimir el valor real de estas alternativas o, eventualmente, de compararlas con un nuevo agente que supuestamente romperá la equiponderación en su favor.

Aunque no es parte reconocida de la discusión sobre equiponderación, conviene introducir una distinción que facilite el análisis posterior. Si las opiniones terapéuticas coexisten indiferentemente, es decir, los partidarios de una alternativa reconocen la validez equivalente de otros modos de proceder, habrá una situación de equiponderación sustentable, que no requiere ser alterada porque los pacientes de uno y otro grupo reciben beneficios comparables. Si, en cambio, se da una situación en que los defensores de una alternativa están en profundo desacuerdo con los demás, se tiene una equiponderación - ya que ningún grupo tiene argumentos mejores que el otro - pero de tipo intolerable o insostenible, pues estando en contradicción, alguna de las alternativas está equivocada al recomendar tratamientos inadecuados o lesivos. La tercera variante es la equiponderación inestable que ocurre cuando se propone investigar un agente terapéutico cuya eventual eficacia desmantelaría la equiponderación existente, lo cual solo se justifica si el nuevo método promete ventajas tan substanciales que rompieran el equilibrio actual. Por último, existe la equiponderación intencionada, cuando frente a una terapia eficaz y establecida se busca por diversas razones, generalmente de orden mercantil, introducir una alternativa de similar efectividad. Si esa fue la razón de ensayar dosis reducidas de AZT en embarazadas HIV positivo, habría sido aceptable si se hubiese comparado con el reconocidamente óptimo protocolo 076. A despecho de la estrategia de comparar contra tratamiento cero para obtener regularmente resultados positivos, lo terapéuticamente útil y éticamente correcto no es saber cuánto mejor que cero es un tratamiento, sino si acaso es aceptablemente menos bueno que el óptimo existente.

Si se observa estas diversas formas de equiponderación desde la perspectiva ética de la protección del paciente - como fue la intención originaria de Fried 19 al introducir el concepto -, es posible concluir lo siguiente: (a) la equiponderación sustentable rehúsa o posterga investigaciones en pro de otras prioridades, protegiendo a los pacientes de estudios innecesarios o confirmatorios de lo ya sabido; (b) la equiponderación insostenible, en cambio, re- clama investigación urgente para identificar cuál práctica médica está dañando a los pacientes a pesar de que la insuficiencia de información las hace aparecer todas como equivalentes - la protección de los pacientes exige que se investigue, identifique y elimine la alternativa perniciosa -; (c) la equiponderación desestabilizada es aquella que es cuestionada por un ensayo que presenta una droga nueva, supuestamente superior y que romperá la equiponderación existente - aunque no hay acuerdo cuán mejor debe presuntamente ser la nueva droga para justificar el estudio, los beneficios que promete han de ser más que marginales, para no caer en el grupo de la equiponderación sustentable que estaría siendo cuestionada innecesariamente -; (d) por fin, la equiponderación intencionada es una meta éticamente justificada si se intenta introducir un nuevo agente que posea una eficacia comparable a un tratamiento efectivo probado y existente, siempre que el nuevo agente posea alguna ventaja adicional mejor tolerancia, menor costo. El mero afán de competir en el mercado introduciendo un agente que no se diferencia substancialmente de lo existente, no debiera justificar los costos, esfuerzos y riesgos de investigaciones clínicas.

Señalan los críticos de la equiponderación: "la equiponderación clínica y toda otra equiponderación sólo tienen sentido como requerimiento normativo en ensayos clínicos bajo la suposición que los investigadores tienen una obligación terapéutica con los probandos" pero esa "obligación terapéutica para investigadores...constituye una falacia terapéutica acerca de la ética de estudios clínicos" 12 (p. 25). Es cierto que la función del científico no es la del terapeuta, pero ello no le otorga licencia para dañar a los probandos, cosa que hace si su investigación interfiere con las necesidades terapéuticas de los pacientes-sujetos. El objetivo de la equiponderación no está en reforzar la metódica científica, sino en la protección a los pacientes de ser expuestos como probandos a terapias insuficientes y riesgos innecesarios.

\section{Conflictos pragmáticos}

Nuevamente es en el articulado de Helsinki 2000 donde queda especificado que los probandos deben continuar recibiendo, más allá del término del estudio, los beneficios que la investigación haya revelado, y que la comunidad huésped ha de ser beneficiada por el hecho de haber albergado la investigación. Los beneficios más allá del estudio han sido impugnados por ser demasiado onerosos y, efectivamente, el párrafo merece ser revisado, pero no para re- 
ducir el requerimiento sino que para especificarlo. El artículo 30 será éticamente correcto cuando señale que los beneficios post-investigacionales deben continuar mientras sean médicamente necesarios 22 . No sólo para no caer en peticiones exageradas, sino también para evitar que los probandos queden desprotegidos. La negativa de conceder terapia continuada después de terminado el ensayo se basa en dos argumentos falibles. Por una parte, se dice que en tratamientos prolongados los costos serían muy altos, mas si se trata de una droga efectiva aunque cara, también producirá excelentes ganancias en el mercado que permitirán absorber el tratamiento gratuito a los pacientes-probandos que ayudaron a desarrollarla. Según se dice, otorgar tratamiento continuado sería un deber imperfecto de compasión, lo cual "es especialmente cierto en el marco de la investigación, donde la meta del quehacer es la verdad, no la solución de problemas personales de salud" 23 (p. 1459).

Las tácitas presuposiciones de la cultura científica, identificadas por Merton 24 hace más de 60 años, son la universalidad, la comunidad, el desinterés y el escepticismo que, aun cuando no se han dado históricamente en forma pura, sufrieron una dramática distorsión en los años 80, al punto que, en opinión del editor del prestigioso periódico inglés Lancet, Horton 25 (p. 8), los contratos entre escuelas de medicina e industrias patrocinantes han "fallado en asegurar que los ensayos clínicos se ciñan a prácticas éticas ampliamente aceptadas, como la protección integral de los pacientes incorporados al estudio". El resultado de este proceso es la erosión de valores científicos tradicionales - tales como los principios según los cuales la investigación se inspira en la curiosidad por saber y por el deseo de propulsar el conocimiento científico-, creando una nueva "ciencia post-académica -, una ciencia que busca beneficios económicos inmediatos" 25 (p. 9).

La indagación por la responsabilidad de investigadores clínicos ha resultado en dos posiciones divergentes: la que entiende "al investigador como médico personal y al sujeto de investigación como paciente", y aquella que identifica "a los investigadores como científicos puros y a los sujetos de investigación como meros voluntarios" 26 (p. 27). La primera es teórica, por cuanto la coincidencia del rol de investigador con la de médico tratante en un mismo sujeto-paciente es excepcional, mas deja en claro que el sujeto de investigación no puede quedar desaventajado en sus requerimientos como paciente. De allí que los investigadores no puedan decir que "en investigación clínica, en contraste [con la asistencia médica], los intereses de los investigadores y los de paciente reclutados como sujetos probablemente diverjan..." 12 (p. 21). Esta posición erosiona severamente el requerimiento del mejor tratamiento existente para proteger adecuadamente a los sujetos-pacientes, máxime si ocurren en "ambientes empobrecidos" y cuando se trata de "individuos particularmente vulnerables" 26 (p. 32). Estas exigencias ético-médicas son tanto más válidas en consideración de lo anteriormente señalado, en el sentido que la investigación biomédica contemporánea ha abandonado la nobleza de la indagación desinteresada, a cambio de la ejecución de programas científicos lucrativos, haciendo poco creíble que "[L]a investigación busca conocimiento acerca de enfermedades y sus tratamientos en grupos de pacientes, con la meta última de mejorar el tratamiento médico" 12 (p. 21).

En cuanto a los beneficios solicitados para las comunidades huésped, también han sido resistidos como un requerimiento desacotado que abriría el imaginario a escenarios irreales como solicitar una elevación del nivel de atención médica o ayuda para mejorar las condiciones socioeconómicas de la población. El tema es abordado con característica vaguedad en la pauta 10 de CIOMS 10 (p. 56): “cualquier intervención o producto desarrollado, o conocimiento generado, estará disponible razonablemente para beneficio de aquella población o comunidad", donde el recurso a lo razonable permite soslayar compromisos vinculantes de cobertura y oportunidad de los beneficios a otorgar. También el artículo 30 de la Declaración de Helsinki requiere mayor especificación en este aspecto, buscando un planteamiento en que probandos y la comunidad-huésped se beneficien concretamente de los resultados de la investigación recibiendo acceso privilegiado o financieramente subvencionado a los productos del estudio. En algunos casos se ha sugerido que la comunidad participe de las eventuales ganancias del producto cuando llegue al mercado, pero no existen fórmulas adecuadas para fijar una proporcionalidad ecuánime. La formulación de este requerimiento debe ser solventable para los países patrocinantes, especificada en acuerdos previos, y controlada en su cumplimiento. Se enfrentan poderosos intereses contradictorios que hacen improbable la resolución de estos conflictos 27 , lo cual va en prejuicio de los más desaventajados y fortifica la posición de los poderosos.

La recomendación de otorgar beneficios comunitarios en un marco de disponibilidad razonable es un término flexible que permite pedir en exceso, así como dejar de otorgar, dando apoyo a la desalentadora conclusión de Macklin 27 (p. 259) al término de su discusión del 
tema de beneficios: "en el fondo creo que la armonización de guías éticas para la investigación no es realizable". Es lamentable que estas figuras semánticas permitan concluir que la idea de justicia distributiva empleada en las guías de CIOMS es demasiado vaga y ambigua para ser utilizada en la evaluación moral de estudios clínicos 28. Estos argumentos olvidan que "la justicia social ha de otorgar significado primordial al destino final de la distribución de recursos entre individuos y sólo secundariamente a los mecanismos utilizados para una tal distribución" 29 (p. 105). El texto invocado cita, a su vez, a Elster, 1989, apud Miller 29 (p. 105): "no debe ponerse el carro de la justicia procedimental por delante del caballo de la justicia substantiva". Para una ética aplicada, eso significa que los esquemas de justicia han de determinar a los individuos o grupos entre quienes se distribuye y especificar cuál es el bien a distribuir. Y eso, en el contexto de una empresa de investigación, es perfectamente delimitable.

\section{Conclusiones}

Con algunas notables excepciones, ha sido la tendencia general de investigadores y bioeticistas del primer mundo respetar una ética de máximos para sus propios países y recurrir a argumentos frágiles e impugnables para defender una ética más flexible y reducida, inaceptable en sus países de origen, para la investigación con seres humanos en naciones cuya pobreza los hace susceptibles a explotación y daño. Es profundamente inquietante que la polémica en torno a las diversas interpretaciones de la ética de investigación con seres humanos se desarrolle a objeto de justificar lo que explícitamente se viene denominando el doble estándar ético, diseñado para hacer "permisible en algunas circunstancias de proveer a los participantes en investigaciones un cuidado inferior al mejor cuidado universal" 4 (p. 190) puesto que se habría logrado consensuar "una posición que, no siendo un estándar ético óptimo, al menos no es claramente inmoral" 4 (p. 192).

La argumentación configura el doble estándar en forma de una ética aspiracional o ética de máximos 27, válida según muchos para las investigaciones realizadas en países el primer mundo, y una ética pragmática, utilizada por los mismos gestores - instituciones, patrocinantes, investigadores - cuando efectúan estudios en el tercer mundo donde las circunstancias sociales y materiales autorizarían la aplicación de exigencias éticas más laxas, bajo el amparo de eufemismos como "diferentes", que no implicaría "más bajas" 3, aunque prontamente se indica que las “normativas CIOMS proveen la más clara afirmación de la necesidad de una razón científicamente válida para utilizar un estándar más bajo de cuidados" 4 (p. 191). Desde allí, los países patrocinantes se han sentido autorizados para cuestionar la competencia de los comités locales de bioética en investigación, de reducir las exigencias del consentimiento informado, de flexibilizar las condiciones de reclutamiento de probandos y, en general, de aplicar métodos de estudio que en sus propios países son éticamente reprobados. Se lee que "muchos comités de países en desarrollo carecen de experiencia, otros hacen un trabajo menos que adecuado para proteger los derechos y el bienestar de sujetos de investigación" 27 (p. 57-8). En vista de la "profundamente arraigada y extensa corrupción en países en desarrollo...es poco lo que investigadores bienintencionados pueden hacer por su cuenta cuando se embarcan en investigaciones destinadas a mejorar las condiciones de salud de poblaciones en países pobres en recursos" 27 (p. 139).

La ética de los países desarrollados está impregnada de conceptos que respaldan el doble estándar, y varios bioeticistas se han dejado tentar a desarrollar argumentos que lo apoyan 4. También existen diversas iniciativas académicas provenientes del primer mundo por impartir en países menos desarrollados, también en Latinoamérica, cursos de capacitación en ética de la investigación: "el Centro Internacional Fogarty del NIH, por ejemplo ha iniciado un programa que provee a representantes de países en desarrollo de entrenamiento en ética, particularmente en ética de investigación Norteamericana ["American" en el original]. Este entrenamiento no garantiza que los comités locales de ética se preocuparán más de proteger a sus conciudadanos que de cooperar con investigadores foráneos sólidamente financiados" 13 (p. 64).

La tarea de la bioética latinoamericana no puede consistir en polemizar en terreno foráneo con fuerzas que poseen todas las ventajas estrategias de un mundo académico potente. Quedan muchos temas por abordar, como paternalismo, explotación, los conceptos de autonomía y consentimiento informado, atingencia local de estudios, participación en patentes etc. Nuestro mandato es de reforzar localmente la resistencia a la colonización pragmática y a los intentos de minar la vocación de todo discurso ético de ser universalmente válido y adoptado por las diversas culturas según sus contextos valorativos, sin permitir discriminaciones que propongan actitudes de segundo nivel ético para quienes son materialmente desposeídos y socialmente marginados. 


\section{Resumen}

Desde la Declaración de Helsinki 2000, se intensificó y amplió un conflictivo debate que ha cuestionado muchos aspectos de la ética de investigación con seres humanos. La polémica incluye el uso de placebos, la aplicación del concepto de equiponderación, la distinción entre ética de investigación y ética clínica, así como la diferencia entre ensayos terapéuticos y no terapéuticos. El presente artículo insiste en fortalecer todos los argumentos, parámetros y estrategias de investigación que sean necesarios para proteger a los paciente-probandos, ante todo el mantenimiento de la mejor terapia existente, el rechazo del uso de placebos, la aplica-

\section{Referencias}

1. Brody BA. The ethics of biomedical research. New York/Oxford: Oxford University Press; 1998.

2. Hossne WS. Poder e injustiça na pesquisa com seres humanos. In: Garrafa V, Pessini L, organizadores. Bioética: poder e injustiça. São Paulo: Edições Loyola; 2003. p. 271-86.

3. Macklin R. After Helsinki: unresolved issues in international research. Kennedy Inst Ethics J 2001; 11:17-36.

4. Lie RK, Emanuel E, Grady C, Wendler D. The standard of care debate: the Declaration of Helsinki. J Med Ethics 2004; 30:190-3.

5. Schüklenk U. The standard of care debate: against the myth of an "international consensus opinion". J Med Ethics 2004; 30:194-7.

6. Diniz D, Corrêa M. Declaração de Helsinki: relativismo e vulnerabilidade. Cad Saúde Pública 2001; 17:679-88.

7. Garrafa V, Prado MM. Mudanças na Declaração de Helsinki: fundamentalismo econômico, imperialismo ético e controle social. Cad Saúde Pública $2001 ; 17: 1489-96$.

8. Garrafa V, Porto D. Bioética, poder e injustiça: por uma ética d intervenção. In: Garrafa V, Pessini L, organizadores. Bioética: poder e injustiça. São Paulo: Edições Loyola; 2003. p. 35-44.

9. Greco DB. Poder e injustiça na pesquisa envolvendo seres humanos. In: Garrafa V, Pessini L, organizadores. Bioética: poder e injustiça. São Paulo: Edições Loyola; 2003. p. 257-69.

10. Council for International Organizations of Medical Sciences. Pautas éticas internacionales para la investigación biomédica en seres humanos. Geneva: Council for International Organizations of Medical Sciences; 2002

11. Hottois G, Missa JN. Nouvelle encyclopédie de bioéhique. Bruxelles: De Boeck \& Larcier; 2001.

12. Miller FG, Brody H. A critique of clinical equipoise. Hastings Cent Rep 2003; 33:19-28.

13. Rothman D. The shame of medical research. New York Ver Books 2000; 47:60-4.

14. Lie RK. Research ethics and evidence based medicine. J Med Ethics 2004; 30:122-5. ción de la equiponderación, la preservación de requerimientos terapéuticos más allá del estudio y la compensación a comunidades-huésped en la medida que ellas hayan facilitado y apoyado la investigación. La propuesta de establecer un doble estándar de ética de investigación en seres humanos, una de máximos para países desarrollados y otra de mínimos pragmáticos a aplicar en países de desarrollo precario, debe ser rechazada por contravenir las intenciones de universalizabilidad de toda prescripción ética.

Ética en Investigación; Declaración de Helsinki; Placebos

15. Angell M. The ethics of clinical research in the Third World. N Engl J Med 1997; 337:847-9.

16. Crouch RA, Arras JD. AZT trials and tribulations. Hastings Cent Rep 1998; 28:26-34.

17. Lurie P, Wolfe SM. Unethical trials of interventions to reduce perinatal transmission of the human immunodeficiency virus in developing countries. N Engl J Med 1997; 337:853-6.

18. Clark PA. AIDS research in developing countries: do the ends justify the means? Med Sci Monit 2002; 8:ED5-16.

19. Fried C. Medical experimentation: personal integrity and social policy. Amsterdam: North-Holland Publishing; 1974.

20. Freedman B. Equipoise and the ethics of clinical research. N Engl J Med 1987; 317:141-5.

21. Menikoff J. Equipoise: beyond rehabilitation? Kennedy Inst Ethics J 2003; 13:347-51.

22. Anonymous. Dismantling the Helsinki Declaration [Editorial]. CMAJ 2003; 169:997-9.

23. Lackey DP. Clinical research in developing countries: recent moral arguments. Cad Saúde Pública 2002; 18:1455-61.

24. Merton RK. Science and the social order. Philos Sci 1938; 5:321-37.

25. Horton R. The dawn of McScience. New York Rev Books 2004; 11:7-9.

26. Richardson HS, Belsky L. The ancillary-care responsibilities of medical research. Hastings Cent Rep 2004; 34:25-33.

27. Macklin R. Double standards in medical research in developing countries. Cambridge: Cambridge University Press; 2004.

28. Cooley DR. Distributive justice and clinical trials in the third world. Theor Med Bioeth 2001; 22:151 67.

29. Miller D. Principles of social justice. Cambridge: Harvard University Press; 1999.

Recibido el 23/Jun/2004

Versión final presentada el 23/Nov/2004

Aprobado el 07/Dic/2004 